Supporting Information for JACS Submission:

\title{
An IR Study of Protonation Changes Associated with Heme-Heme Electron Transfer in Bovine Cytochrome $c$ Oxidase
}

\author{
Masayo Iwaki and Peter R. Rich \\ Glynn Laboratory of Bioenergetics, Department of Biology, University College London, Gower \\ Street, London WC1E 6BT, U.K. \\ E-mail: PRR@UCL.AC.UK
}

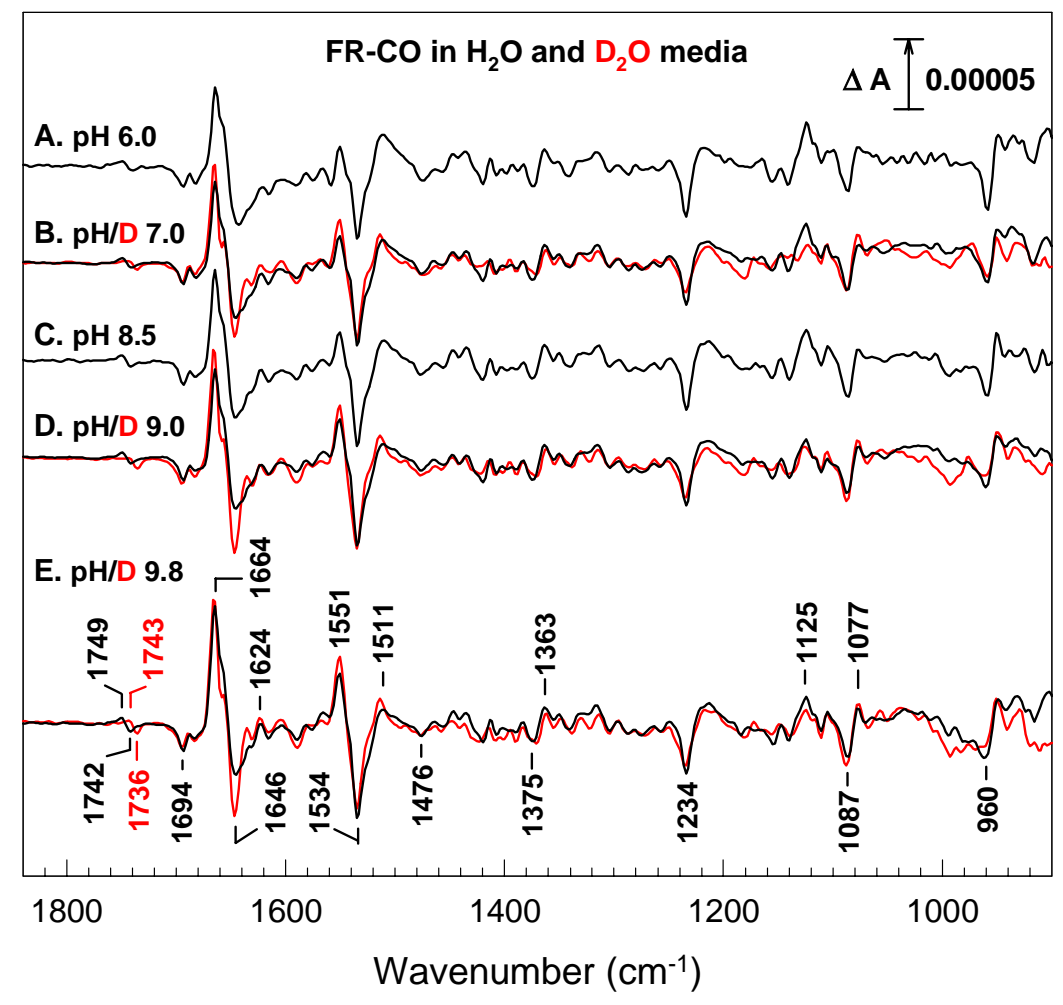

Figure S1. ATR-FTIR spectra of CO-photolysis of the FR-CO form of $\mathrm{CcO}$. Light minus dark difference ATR-FTIR spectra were measured in FR-CO form of bovine $\mathrm{C} c \mathrm{O}$ at pH 6.0 (trace A), 7.0 (trace B, same data in Fig. 2A), 8.5 (trace C), 9.0 (trace D) and 9.8 (trace E) in $\mathrm{H}_{2} \mathrm{O}$ media (black) and at pD 7.0 (trace B, same data in Fig. 2A), 9.0 (trace D) and 9.8 (trace E) in $\mathrm{D}_{2} \mathrm{O}$ media (red). Typically, the final raw spectra were obtained from total 1,000-2,500 dark/light cycles with 100 interferograms each. The broad heating baseline has been subtracted. A amplitudes of spectra are normalized to give the same intensities of negative band of bound CO. Since the spectra are essentially $\mathrm{pH}(\mathrm{pD})$-independent, peak/trough labellings are shown only for data at $\mathrm{pH} / \mathrm{D} 9.8$. 


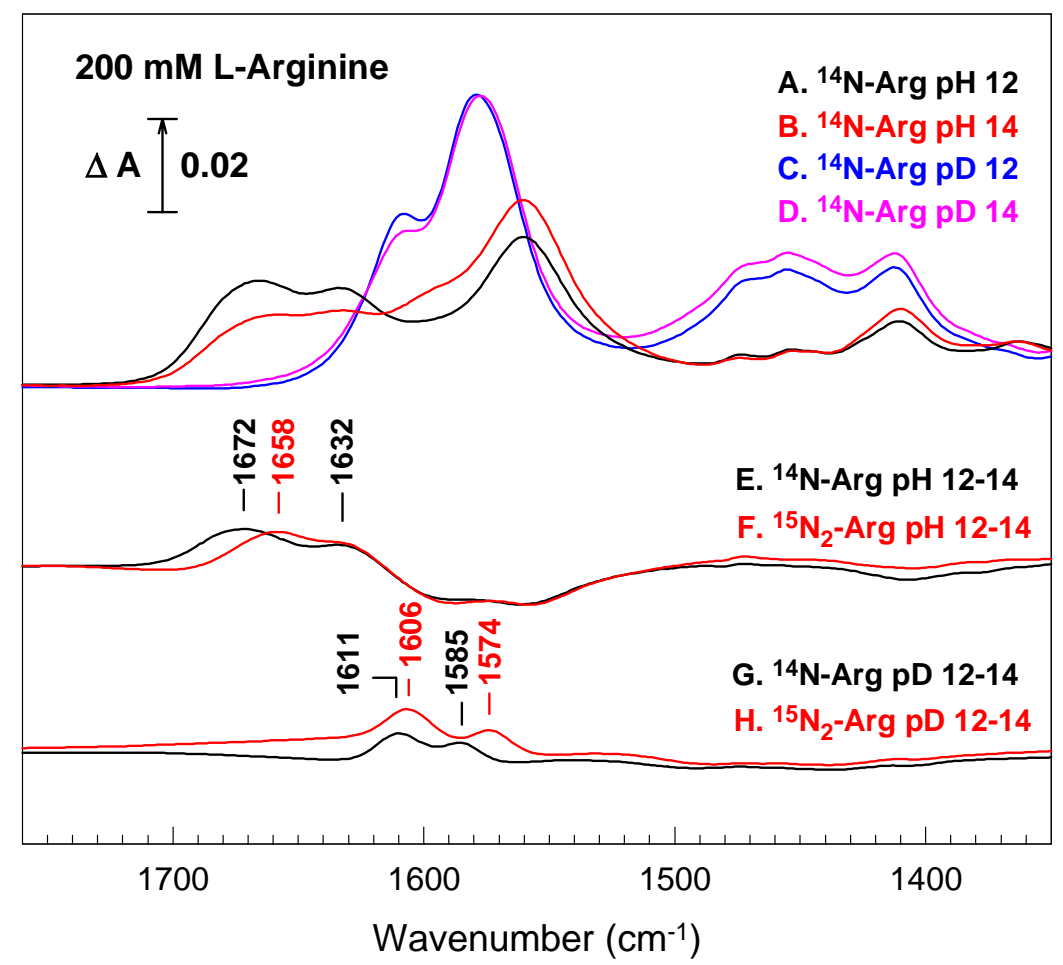

Figure S2. ATR-FTIR spectra of L-arginine. $200 \mathrm{mM}$ solutions of unlabelled and ${ }^{15} \mathrm{~N}_{2}$-[guanidinium]labelled L-arginine were prepared in $\mathrm{H}_{2} \mathrm{O}$ or $\mathrm{D}_{2} \mathrm{O}$ at pH/D 12 or 14 by adjustment with KOH. 5-10 $\mu \mathrm{L}$ aliquots were placed on the ATR prism and the spectra were averaged from 1,000 interferograms versus a clean prism background. The solvent contribution had been subtracted. Absolute ATR-FTIR spectra of unlabelled L-arginine at pH 12 (trace A, black) and 14 (trace $\mathrm{B}$, red) in $\mathrm{H}_{2} \mathrm{O}$ and at pD 12 (trace C, blue) and 14 (trace D, pink) in $\mathrm{D}_{2} \mathrm{O}$. The difference between pH/D $12-\mathrm{pH} / \mathrm{D} 14$ are: ${ }^{14} \mathrm{~N}_{2}-\operatorname{Arg}$ in $\mathrm{H}_{2} \mathrm{O}$, black trace $\mathrm{E}$ (i.e. A minus B); ${ }^{15} \mathrm{~N}_{2}$-Arg in $\mathrm{H}_{2} \mathrm{O}$, red trace $\mathrm{F} ;{ }^{14} \mathrm{~N}_{2}$-Arg in $\mathrm{D}_{2} \mathrm{O}$, black trace $\mathrm{G}$ (i.e. $\mathrm{C}$ minus D); ${ }^{15} \mathrm{~N}_{2}$-Arg in $\mathrm{D}_{2} \mathrm{O}$, red trace $\mathrm{H} .{ }^{15} \mathrm{~N}_{2}$-labelled arginine was a kind gift from Prof. W. John Ingledew. 